\title{
Treatment for Peritoneal Metastasis of Patients With Colorectal Cancer
}

\author{
Young Jin Kim ${ }^{1}$, Chang Hyun Kim² \\ ${ }^{1}$ Department of Surgery, Seokjeong Wellpark Hospital, Gochang; ${ }^{2}$ Division of Colorectal Surgery, Department of Surgery, Chonnam National \\ University Hwasun Hospital, Chonnam National University Medical School, Hwasun, Korea
}

From the perspective of survival outcomes, the cancer survival of colorectal cancer (CRC) in the whole stage has improved. Peritoneal metastasis (PM) is found in approximately $8 \%$ to $15 \%$ of patients with CRC, with a poorer prognosis than that associated with other sites of metastases. Randomized controlled trials and up-to-date meta-analyses provide firm evidence that cytoreductive surgery (CRS) plus hyperthermic intraperitoneal chemotherapy (HIPEC) could significantly improve overall survival compared with systemic chemotherapy alone in selected patients with CRC-PM. Practical guidelines recommend that the management of CRC-PM should be led by a multidisciplinary team carried out in experienced centers and consider CRS plus HIPEC for selected patients. In this review, we aim to provide the latest results of land mark studies and an overview of recent insights with regard to the management of CRC-PM.

Keywords: Cytoreduction surgical procedures; Hyperthermic intraperitoneal chemotherapy; Peritoneal metastases; Colorectal cancer

\section{INTRODUCTION}

Globally, colorectal cancer (CRC) is the third most common cancer and second most common cause of cancer-related mortality. In South Korea, the incidence of CRC has increased remarkably over the past decades [1]. From the perspective of survival outcomes, the cancer survival of CRC in the whole stage has improved. The 5-year survival rate of metastatic CRC has improved from $4 \%$ to $12 \%$ over the past 2 or 3 decades, mainly due to the use of intensive chemotherapy $[2,3]$. Peritoneal metastasis $(\mathrm{PM})$ is found in approximately $8 \%$ to $15 \%$ of patients with CRC during the first treatment cycle [4]. Furthermore, $2 \%$ of patients have isolated PM and $11 \%$ have PM with other organ involvement, with a poorer

Received: Oct 25, 2021 - Revised: Nov 15, 2021 - Accepted: Nov 15, 2021 Correspondence to: Young Jin Kim, M.D., Ph.D.

Department of Surgery, Seokjeong Wellpark Hospital, 147 Seokjeong 2-ro, Gochang-eup, Gochang 56451, Korea

Tel: +82-63-560-1000, Fax: +82-63-560-1163

E-mail: kimyjin@jnu.ac.kr

ORCID: https://orcid.org/0000-0003-1861-8119

(C) 2021 The Korean Society of Coloproctology

This is an open-access article distributed under the terms of the Creative Commons Attribution NonCommercial License (https://creativecommons.org/licenses/by-nc/4.0) which permits unrestricted noncommercial use, distribution, and reproduction in any medium, provided the original work is properly cited. prognosis than that associated with other sites of metastases (shorter overall survival [OS] by 30\%-40\%) [5-7]. The effect of systemic chemotherapy in patients with isolated PM is limited and only slightly improves the median OS to 16.3 months [5].

This indicates that $5 \%$ to $15 \%$ of CRC cases would be included in this group; hence, the surgical treatment of PM is expected to have a significant impact. Moreover, the surgical management of potentially resectable PM has evolved profoundly in the past $2 \mathrm{de}-$ cades. This review highlights the key components of PC, including its biological and clinical characteristics, recent treatment strategies, future studies, and our experiences with PM of CRC.

\section{BIOLOGY OF PERITONEAL METASTASIS}

Primary cancers of the peritoneum are rare, with an incidence rate of 6 to 7 per million individuals [8]. However, secondary tumors of the peritoneum are more common; in particular, for CRC, PM represents the second most common site of metastasis after the liver [9]. PM can be explained by different mechanisms currently accepted in this field, including the hematogenous or lymphatic spread of CRC. Surface intrusion and migration, invasion into the submesothelial tissue, and spontaneous or traumatic (associated with previous surgeries) perforation by tumor cells that 
are potentially adapted or enabled by mutations. Different cell signaling pathways linked to each step of peritoneal dissemination imply several molecules: (i) tumor shedding and detachment (Ecadherin and epithelial-to-mesenchymal transition); (ii) transport within the peritoneum (actin microfilament system); (iii) dissemination (intercellular adhesion molecule 1), vascular adhesion molecule 1, tumor cell receptors (cluster of differentiation 44), cytokines (tumor necrosis factor alpha, interleukin-1 beta, and interleukin-1 gamma); (iv) invasion (metalloproteinases and integrins); and (v) proliferation and angiogenesis (epidermal growth factor receptor, epidermal growth factor, tumor growth factor $\alpha$, insulinlike growth factor-1, hypoxia-inducible factor, vascular endothelial growth factor [VEGF], and VEGF receptor) [10].

In 2015, a collaborative study group that had previously developed a methodology for CRC classification based on gene expression was established [11]. They developed the consensus molecular subtype (CMS) classification. This classification is composed of 4 subtypes (CMS1-4), each of which has a specific biological behavior and subsequent implications for optimal treatment and prognosis. Of these, the CMS4 subtype, also known as the mesenchymal subtype, is considered the most difficult to treat and has the worst prognosis. This subtype is characterized by stromal infiltration, overexpression of extracellular matrix proteins, and high mixture with non-cancer cells. In terms of PM, a recent study has suggested that the CMS4 subtype is frequently diagnosed in primary tumors of patients presenting with PM (60\%) [12].

An attempt to determine the accurate molecular mechanisms associated with PM is necessary to develop selective biomarkers and targeted agents.

\section{DIAGNOSIS OF PERITONEAL METASTASIS}

The risk factors for developing PM of CRC are the stage of disease, intra-abdominal colon location, infiltrative carcinoma, mucinous adenocarcinoma, and age younger than 70 to 75 years [13].

The goal of the preoperative assessment of patients with PM of $\mathrm{CRC}$ is to select potential candidates for complete resection. The gold standard for diagnosing PM is positive peritoneal cytology [14]. Based on the sensitivity of computed tomography (CT) as a reference imaging modality, the sensitivity and specificity for detecting PM are $83 \%$ (95\% confidence interval [CI], 79\%-86\%) and $86 \%$ (95\% CI, 82\%-89\%), respectively [15]. However, the sensitivity for small lesions $(<5 \mathrm{~mm})$ is reduced to $43 \%$. In particular, sensitivity and specificity were particularly limited in the mesentery, which is a crucial region for achieving complete tumor resection [16]. In addition, when a small PM lesion scallops to the liver surface, it is difficult to differentiate from subcapsular liver metastasis, given that each clinical situation can considerably affect the treatment strategy. In this regard, magnetic resonance imaging (MRI) is better and should be considered to avoid unexpected clinical situations. However, the generalizability of MRI findings in the workup of PM has yet to be validated. Despite technical ad- vancements, MRI is more operator-dependent with a long learning curve. The spatial resolution of MRI remains inferior to that of CT for the elucidation of PM lesions. However, MRI allows for the differentiation of ascites from solid tumor deposits and performs well for the detection of mucinous tumors. In addition, diffusion-weighted MRI outperforms other imaging techniques in terms of sensitivity and specificity [17]. The performance of positron emission tomography (PET) alone is inferior to that of CT. However, it can be enhanced when combined with CT (PET-CT). One study has shown that PET-CT is better at predicting peritoneal cancer index (PCI) than CT alone [18].

Surgical exploration is the gold standard technique for the evaluation of PM. The active use of exploratory laparoscopy could reduce unnecessary laparotomy in $40 \%$ of patients [19]. Diagnostic laparoscopy is often used to evaluate occult carcinomatosis or reduce the laparotomy rate in patients for whom cytoreductive surgery (CRS) plus hyperthermic intraperitoneal chemotherapy (HIPEC) may not be possible. Confirmation of the possibility of complete PM resection is a prerequisite condition for HIPEC because of limited intraperitoneal tissue penetration (less than $2.5 \mathrm{~mm}$ ). In particular, involvement of the membranous diaphragm, mesenteric root, pancreas, and porta hepatis should be carefully explored. Digital rectal examinations for the detection of nodules in the pouch of Douglas is one of the most important diagnostic techniques. Complete clinical evaluations of primary and peritoneal tumors and states of other distant metastases should be performed before any treatment. An expert group proposed 5 points for the operative reports of PM as follows [20]: (1) description of lesions quadrant by quadrant and calculation of the PCI; (2) presence or absence of ascites; (3) photos or videos; (4) detailed description of small bowel or mesenteric involvement; and (5) detailed description of right diaphragmatic dome involvement and whether the cupola is mobile or not.

Intraoperative fluorescence imaging is an emerging technology that is helpful in evaluating blood supply in colorectal surgery. Although only a few clinical results are available to date, the potential role of fluorescence imaging in the detection of PM has been tested. Liberale et al. [21] reported that surgery was modified by intraoperative indocyanine green fluorescence imaging in 4 of 14 patients (29\%), in which additional lesions not found using surgical exploration modified the surgery. New molecular fluorescence imaging techniques, such as those using a fluorescent dye attached to bevacizumab, have been actively tested [22]. Further studies are needed to standardize the optimal timing for improving the sensitivity of these techniques and to evaluate its impact on long-term results.

\section{TREATMENT OF COLORECTAL CANCER- PERITONEAL METASTASIS}

Currently, the best survival duration reported following only systemic modern chemotherapy and supportive care for PM of color- 
ectal origin is 15.2 to 23.4 months [5, 23]. To date, CRC-PM has been considered an incurable disease, and palliative measures have remained the choice of management. However, CRC-PM is now gradually regarded as regional tumor progression, suitable for radical therapeutic strategies with CRS and HIPEC, which are likely to achieve prominent clinical benefits in selected patients, and was introduced and popularized for the treatment of PM of appendiceal neoplasms by Sugarbaker [24]. Since then, it has been expanded to cases of CRC patients, the value of CRS with HIPEC in CRC-PM has been studied in many single-institute studies that reported better survival outcomes compared with systemic chemotherapy alone [25-29].

\section{COLORECTAL CANCER WITH HYPERTHERMIC INTRAPERITONEAL CHEMOTHERAPY}

Randomized controlled trials (RCTs) and up-to-date meta-analyses provide firm evidence that CRS plus HIPEC could significantly improve OS compared with systemic chemotherapy alone in selected patients with CRC-PM. In 2003, Verwaal et al. [30] showed improved OS in patients treated with CRS plus mitomycin C-based HIPEC and the same adjuvant chemotherapy using 5-fluoroura$\mathrm{cil} /$ leucovorin (5-FU): 12.6 months in the standard treatment group vs. 22.3 months in the CRS plus HIPEC group $(\mathrm{P}=0.028)$. Specifically, in patients amenable to complete resection, CRS plus HIPEC showed a 5-year OS of 54\%. They updated the median follow-up period in their initial study from 21.6 months to 8 years and strengthened their previous results [31]. This study also showed that the prognosis of CRC-PM after CRS plus HIPEC is related to the completeness of surgical resection. However, some critiques of this RCT remain. The systemic chemotherapy used in the study (5-FU/leucovorin) was regarded as obsolete. In addition, approximately $17 \%$ (18 of 105) of the patients in the trial had appendiceal adenocarcinoma. A relatively high mortality rate in the CRS plus HIPEC arm was reported (8\%). Nevertheless, even with these striking results, this study could not lead to an evolutionary paradigm shift in the treatment of CRC-PM, which has progressed to slow implementation in highly experienced centers and gradually accumulating evidence.

Most importantly, the role of CRS and proportion of the benefit that results from HIPEC have been questioned in the era of increasingly more advanced systemic chemotherapy for metastatic CRC. In terms of the role of CRS after the introduction of FOLFOX (folinic acid, fluorouracil, and oxaliplatin) treatment, 2 case-control studies compared outcomes in patients undergoing CRS with those in matched patients treated with systemic therapy alone. They found a superior OS in surgically treated patients (63 vs. 24 months and 35 vs. 17 months, respectively) [32, 33]. In addition, a single-institution RCT (closed early) compared surgery and early postoperative intraperitoneal chemotherapy (EPIC) using 5-FU as an adjuvant setting with 6 months of systemic FOLFOX treatment alone.
Despite early termination, this study showed that the median OS durations were 25 months in the surgery arm and 18 months in the chemotherapy arm $(\mathrm{P}=0.04)$, confirming the superior survival to that of systemic chemotherapy alone even in the era of FOLFOX treatment [34]. Second, there was a concern that the contribution of intraperitoneal chemotherapy (either form of HIPEC or EPIC) would exist. Recently, an RCT that aimed to assess the specific contribution of adding HIPEC to CRS in comparison with CRS alone did not show a survival benefit for HIPEC. A total of 265 patients were enrolled, and all patients received systemic chemotherapy. Patients in whom complete macroscopic resection or surgical resection with less than $1-\mathrm{mm}$ residual tumor tissue was completed were randomly assigned (1:1) to CRS with or without oxaliplatin-based HIPEC. After a median follow-up duration of 64 months, the median OS durations were 42 months in the CRS plus HIPEC arm and 41 months in the CRS arm $(\mathrm{P}=0.99)$ [35]. The authors failed to show evidence of an improvement in OS with the addition of HIPEC compared to that of CRS alone. It was the first report of the specific role of HIPEC, which was contrary to traditional beliefs. In addition, the complication rate was also higher in the HIPEC group. The authors suggested that CRS alone should be the cornerstone of treatment strategies for CRC-PM. However, many criticisms of this study suggest that HIPEC should not be abandoned prematurely. First, the enrolled patients were heavily pretreated, as 219 of 265 (83\%) patients received preoperative chemotherapy (of whom, 127 [58\%] received 6 or more cycles), probably FOLFOX, with a long interval between the diagnoses of CRS-PM and CRS (median intervals of 149 days in the CRS plus HIPEC group and 164 days in the CRS group). As stated by the authors, these selected patients might have been good responders to preoperative chemotherapy and had fewer additional survival benefits of adding oxaliplatin-based HIPEC. Second, the use of mitomycin C-based rather than oxaliplatin-based HIPEC is unlikely to have resulted in a different outcome from those of this study. Regarding the status corresponding to the biology of CRC-PM, the majority of CRC-PMs (75\%) were classified as CMS4. In addition, this subtype is less sensitive to oxaliplatin-based chemotherapy than the other subtypes [36]. The finding that CMS4 is the predominant subtype in CRC-PM, in addition to its resistance to oxaliplatin, could be another reason why trials using oxaliplatin-based HIPEC have failed to show survival benefits.

\section{PRESSURIZED INTRAPERITONEAL AEROSOL CHEMOTHERAPY}

The major limitations of intraperitoneal chemotherapy with lavage are low tissue penetration and inhomogeneous distribution. Instead of distributing the chemotherapeutic agents in the peritoneal cavity in the form of a fluid solution, in pressurized intraperitoneal aerosol chemotherapy (PIPAC), the drug solution is aerosolized. Aerosols consist of 2 phases; fluid (droplets) and gaseous. Gases are distributed homogeneously within a closed space; there- 
fore, homogenous distribution of the drug throughout the entire abdominal cavity is more prominent than with a fluid solution. In addition to an even distribution, a relatively high pressure is created between the peritoneal cavity and interstitium of the peritoneal tumor. Consequently, the biodistribution of substances in the peritoneum is improved. PIPAC is a novel approach in the palliative treatment of non-resectable PM based on laparoscopically administered aerosolized chemotherapy into the hyperbaric capnoperitoneum. This concept of "therapeutic pneumoperitoneum" was first described in 2000 [37]. The authors proposed that this concept could be applied as a promising platform for the treatment of peritoneal cancer, prevention of port-site recurrences, immunomodulation, analgesia, peritonitis, and prevention of postoperative adhesions. However, due to technical limitations, it was impossible to immediately implement this concept clinically; hence, a new device to overcome these technical problems was developed a decade later [38]. The drug distributions applied by PIPAC and peritoneal lavage were compared in an ex vivo study. The authors demonstrated that the aerosolized test substance (Dbait) was biologically active up to $1 \mathrm{~mm}$ in depth and showed more activity in tumor nodules than in bare peritoneum, whereas no activity was found in the conventional lavage samples [39]. However, conflicting results have been found in recent studies on candidate molecular distribution and tissue concentrations of PIPAC. To date, no clear indications for PIPAC in the treatment of CRC-PM have been reported. Although trials on the use of PIPAC-directed treatment as prophylaxis for intraperitoneal metastases are currently ongoing, PIPAC is considered a palliative treatment. Moreover, it is currently regarded as an alternative treatment for patients who are ineligible for radical treatment. Solass et al. [39] reported a multicenter, single-arm, phase 2 trial that aimed to assess the safety and antitumor activity of oxaliplatin-based PIPAC in patients with unresectable CRC-PM. Non-negligible major adverse events (15\%) occurred, and minor adverse events were common. Despite the considerable response of biochemical, pathological, cytological, and ascites amounts, no radiological or clinically relevant macroscopic responses were observed. Currently, data regarding the feasibility, toxicity, quality of life, and assessment tools for quantifying treatment response are lacking; therefore, future studies should address these issues.

\section{PREVENTION OR EARLY DETECTION OF COLORECTAL CANCER-PERITONEAL METASTASIS}

As described above, PM remains difficult to detect, both clinically and radiologically. T4 disease, tumor perforation, ovarian metastases, and positive ascites cytology are well-known risk factors for recurrence in the peritoneum after index surgery for CRC [23, 40]. Patients with a high probability of PM can be categorized into 2 distinct approaches: prophylactic HIPEC and invasive surveillance, such as systematic second-look surgery.
The role of prophylactic HIPEC was evaluated in the COLOPEC trial [41]. This study enrolled 204 patients and randomly assigned patients with either T4 or perforated colon cancer to either adjuvant HIPEC with standard systemic chemotherapy or surveillance with systemic chemotherapy. They aimed to evaluate peritoneal recurrence-free survival (PFS) at 18 months, as determined based on $\mathrm{CT}$ and/or laparoscopy. In the intention-to-treat analysis, there was no difference in PFS at 18 months: 80.9\% (95\% CI, 73.3-88.5) in the experimental group vs. $76.2 \%$ (95\% CI, $68.0-84.4)$ in the control group $(\mathrm{P}=0.28)$. The authors did not recommend the routine use of adjuvant HIPEC with oxaliplatin in patients with $\mathrm{T} 4$ or perforated colon cancer. Some investigators have similarly proposed the concept of "systematic second-look surgery and HIPEC" in the absence of any signs of recurrent disease in highrisk patients. The PROPHYLOCHIP trial randomized patients to either second-look surgery plus prophylactic HIPEC or standard follow-up after 6 months of systemic chemotherapy. After a median follow-up of 51 months, the 3-year disease-free survival rates were $53 \%$ in the standard follow-up arm vs. $44 \%$ in the secondlook surgery arm $(\mathrm{P}=0.82)$. The OS rates did not vary at 3 years ( $80 \%$ vs. $79 \%$ ) and 5 years ( $72 \%$ vs. $68 \%$ ) [42]. These 2 trials, which both failed to demonstrate their primary endpoint, indicated that "proactive" oxaliplatin-based HIPEC can no longer be recommended. However, possible explanations for these negative results originate from several factors: inadequate intervention timing, relatively short use of oxaliplatin-based 30-minute HIPEC, and ineffective inclusion criteria to identify a population of patients with colon cancer with a sufficiently high risk of PM. Hence, future studies should address these questions.

\section{PROCEDURE OF CYTOREDUCTIVE SURGERY AND HYPERTHERMIC INTRAPERITONEAL CHEMOTHERAPY}

The 6 peritonectomy procedures originally include (1) greater omentectomy-splenectomy, (2) left upper quadrant peritonectomy, (3) right upper quadrant peritonectomy, (4) lesser omentectomy-cholecystectomy with stripping of the omental bursa, (5) pelvic peritonectomy with sleeve resection of the sigmoid colon, and (6) antrectomy [24]. However, splenectomy and antrectomy are not always recommended, and nodules at the serosal surface are stripped using Metzenbaum scissors. We performed en bloc peritonectomy of the parietal peritoneum and pelvic peritoneum through a long midline incision from the xiphoid process to the pubic bone to achieve completeness of cytoreduction (CCR) of CRC-PM in visceral organs, such as the stomach, colon, and intrapelvic organs simultaneously (Fig. 1).

On completion of the surgery, the CCR score was recorded: CCR0 , no residual macroscopic tumor; CCR-1, residual tumor deposits $<2.5 \mathrm{~mm}$ in diameter; and CCR-2, residual tumor deposits $>2.5$ $\mathrm{mm}$ in diameter [43]. After completing CRS (CCR-0 or CCR-1), HIPEC was performed using the open coliseum technique with 4 

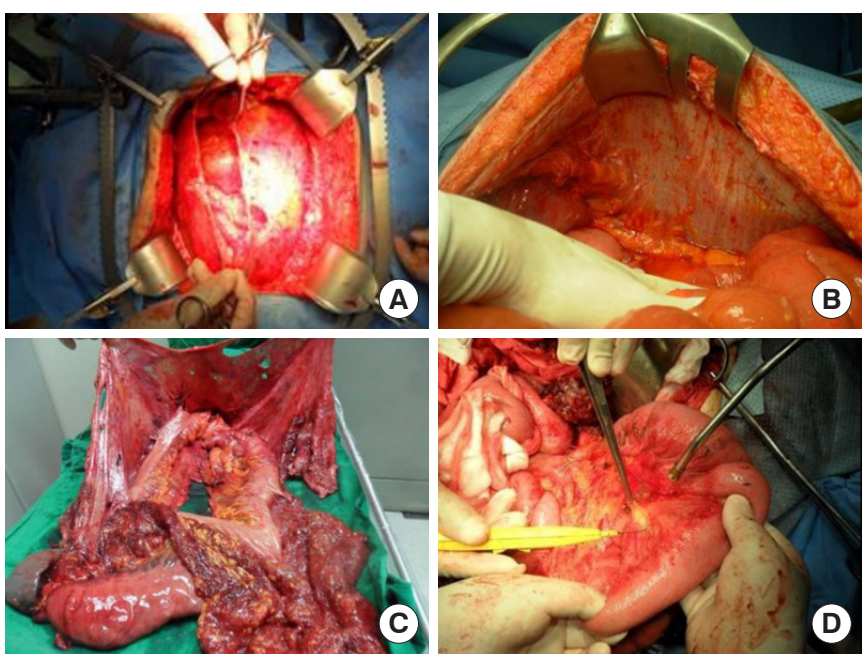

Fig. 1. (A) Pelvic peritonectomy. (B) Peritonectomy of left upper quadrant. (C) En bloc peritonectomy with stomach, spleen, and colon except for small bowel. (D) Visceral peritonectomy of small bowel mesentery. Clinical images from Chonnam National University Hwasun Hospital.

L of physiological saline $(0.9 \%)$ as the perfusate. The target temperature was $42.5^{\circ} \mathrm{C}$ to $43.5^{\circ} \mathrm{C}$, and the treatment time was 30 to 60 minutes.

Maximal CRS was performed to remove the primary tumor with acceptable margins and any involved adjacent tissues and organs, regional lymph nodes, and peritonectomy. Unresectable tumors were cauterized using a ball-tipped electrosurgical device. The CCR score was evaluated before HIPEC [44]. Subsequently, HIPEC was implemented using the open Colosseum or closed techniques. We used the latter, and the inlet and outlet tubes were inserted through 12-mm balloon trocars. Thermosensor catheters were introduced beside the trocars.

As described, HIPEC can be performed in a closed or open abdominal cavity. The open technique allows for continuous manipulation during the procedure, whereas the closed technique may cause reduced drug exposure in the medical team. Many hypothetical advantages and disadvantages have been assumed, but these have not been clinically proven. One literature survey revealed that of the 171 HIPEC studies, the open method was investigated in $44 \%$, whereas the closed technique was used in $25 \%$. The remainder of the publications might cover many cases wherein the technique used was not reported [45].

Currently, there are 3 main types of intraperitoneal chemotherapy: HIPEC, sequential postoperative intraperitoneal chemotherapy (SPIC) [46], and EPIC [25]. HIPEC and other modalities can be combined [47]. In HIPEC, the temperatures used are above physiological levels of $37^{\circ} \mathrm{C}$ (usually $41^{\circ} \mathrm{C}-43^{\circ} \mathrm{C}$; temperatures above $44^{\circ} \mathrm{C}$ cause apoptosis in normal cells). Both EPIC and SPIC are postoperative normothermic treatments. EPIC is an intraperitoneal chemotherapy treatment administered in the immediate postoperative period, usually 4 to 6 days after surgery, whereas SPIC is an adjuvant treatment administered over 6 months. EPIC and SPIC were evaluated in RCTs; however, these were conducted more than 30 years prior or have not been adequately addressed because they had to be terminated prematurely due to slow recruitment $[34,48]$. Although some case-control studies have demonstrated that HIPEC combined with EPIC is associated with improved survival, an increasing trend toward performing CRS plus oxaliplatin-based HIPEC makes EPIC a less preferred option [46, 49]. Moreover, the additive tumoricidal effect of hyperthermia, which is mainly used in landmark RCTs, lack of robust data due to early termination of prospective studies using EPIC or SPIC, increased concern for complications caused by long-term exposure to cytotoxic drugs, and short intraoperative chemotherapy duration of currently used oxaliplatin-based HIPEC (30 minutes) make HIPEC a more preferable modality than EPIC or SPIC [50, 51]. Nonetheless, the effectiveness of intraoperative intraperitoneal chemotherapy is highly dependent on the presence of chemotherapeutic agents but not on hyperthermia, based on an experimental study [52]. As with many cases in this field, to date, no RCTs have compared EPIC with HIPEC. In this regard, the ICA$\mathrm{RuS}$ trial attempts to address some of these questions; it aims to delineate a survival benefit from heated mitomycin C-based HIPEC for a 100-minute treatment vs. normothermic 5-FU-based EPIC [53].

\section{NEOADJUVANT CHEMOTHERAPY BEFORE CYTOREDUCTIVE SURGERY AND HYPERTHERMIC INTRAPERITONEAL CHEMOTHERAPY}

CRC-PM mostly arises from advanced primary tumors with a high risk of systemic spread. Hypothetically, neoadjuvant systemic therapy may decrease the intraperitoneal tumor load, and perioperative systemic therapy may eradicate systemic micrometastases. In contrast, perioperative systemic chemotherapy can cause toxic effects. For example, it may increase postoperative complications, especially when VEGF inhibitors are added [54, 55]. However, there is currently no consensus regarding this issue both in neoadjuvant and adjuvant settings, in the treatment of CRC-PM in patients undergoing CRS plus HIPEC. Two previous systematic reviews failed to provide firm evidence and concluded that there is no high-level evidence to guide systemic chemotherapy [56, 57]. As a result, the decision to add neoadjuvant and/or adjuvant chemotherapy after CRS plus HIPEC is at the discretion of an expert. Fortunately, some RCTs have enrolled patients. Of these, the recently published COMBATAC trial showed the importance of intention-to-treat analysis in neoadjuvant settings. In the COMBATAC trial [58], patients with CRC-PM and appendiceal PM were included and treated with preoperative systemic polychemotherapy plus cetuximab followed by CRS plus HIPEC and postoperative systemic polychemotherapy plus cetuximab. Although this 
trial was prematurely terminated because of slow accrual, the study provided important insights into the neoadjuvant treatment of CRM-PM on an intention-to-treat basis. It is highly probable that a high proportion of patients would not proceed with CRS plus HIPEC after neoadjuvant systemic chemotherapy. Of the 25 patients who received neoadjuvant treatment, CRS plus HIPEC was administered only in 14 patients. A significant rate of patient dropout during neoadjuvant treatment should be considered, and a selection bias is likely to be entailed. Consequently, the remarkably good survival in the PARODIGE-7 trial [35] could be partially explained.

To address this issue in an RCT, the Dutch CAIRO trial [59] was initiated in 201 and published in 2021. In their randomized phase 2 trial (combined phase 3 trial), 79 patients were randomized to perioperative systemic therapy (experimental arm) or CRS plus HIPEC alone (control arm, open technique with either mitomycin C ( $35 \mathrm{mg} / \mathrm{m}^{2}, 90$ minutes) or oxaliplatin $\left(460 \mathrm{mg} / \mathrm{m}^{2}, 30 \mathrm{~min}-\right.$ utes) with intravenous leucovorin $\left(20 \mathrm{mg} / \mathrm{m}^{2}, 10\right.$ minutes $)$ and 5 -FU ( $400 \mathrm{mg} / \mathrm{m}^{2}, 15$ minutes) according to a local protocol. The trial arms did not differ significantly in terms of the proportions of macroscopic complete CRS (experimental, 89\%; control, 86\%) and major postoperative morbidity (experimental, 22\%; control, $33 \%)$. Accordingly, the authors suggested that perioperative systemic chemotherapy may be feasible and safe for CRC-PM, justifying a phase 3 trial.

\section{INTRAPERITONEAL TARGETED THERAPY}

Intraperitoneal bevacizumab therapy has been theorized to improve outcomes in patients with malignant ascites. VEGF expression decreases in ascites after intraperitoneal bevacizumab administration [60]. Ziv-aflibercept, a recombinant protein that inhibits the VEGF pathway, has a stronger anticancer effect than that of bevacizumab in CRC in animal models. In this regard, intraperitoneal ziv-aflibercept may be a valuable candidate for palliating refractory ascites without causing severe adverse events in patients with CRC-PM [61].

\section{INTRAPERITONEAL IMMUNOTHERAPY}

Intraperitoneal immunotherapy is a promising approach that is currently under active investigation for patients with advanced PM. Advances in cancer immunotherapy over the last 3 decades have yielded state-of-the-art therapies with invaluable potential. Today, multiple treatment strategies involving the utilization of an immune response to tumor cells are available. These include antibody-, T-cell-, and viral-based approaches; each approach has unique challenges and advantages. For this reason, a combinatorial approach to immunotherapy is an attractive and ongoing focus of investigation. For example, in April 2009, catumaxomab was approved for intraperitoneal immunotherapy in Europe, to control malignant ascites in patients with epithelial cell adhesion molecule-positive cancer [62]. Despite early assurance for the management of CRC-PM, further clinical trials are necessary. Nevertheless, intraperitoneal immunotherapy will definitely be a future treatment for CRC-PM.

\section{CONCLUSION}

Once regarded as an untreatable condition with an invariably poor outcome, the introduction of CRS plus HIPEC has changed the perspective for patients with CRC-PM. The most important prognostic factors in the treatment of CRC-PM are PCI and CCR. En bloc peritonectomy can help achieve CCR-0 effectively. However, some critiques of HIPEC have been raised since the remarkable publication of the associated trial. In this regard, we await the results of the PRODIGE-7 trial, which will provide firm evidence to further improve treatment and future studies. However, abandoning the concept of intraperitoneal drug delivery based on this trial alone appears premature. Although complete resection based on state-of-the-art surgery will remain the cornerstone of treatment, the integration of our knowledge on molecular biology, various drug delivery systems such as PIPAC, and the development of new drugs specifically targeting CMS4 will revolutionize treatment in this field. With these changes, regionally targeted therapy may include VEGF inhibitors and intraperitoneal immunotherapy with cytokines, cytotoxic $\mathrm{T}$ cells, and/or natural killer cells; hence, the eradication of immunosuppressive cell therapy is promising.

\section{CONFLICT OF INTEREST}

No potential conflict of interest relevant to this article was reported.

\section{REFERENCES}

1. Hur H, Oh CM, Won YJ, Oh JH, Kim NK. Characteristics and survival of Korean patients with colorectal cancer based on data from the Korea Central Cancer Registry data. Ann Coloproctol 2018;34:212-21.

2. Brouwer NP, Bos AC, Lemmens VE, Tanis PJ, Hugen N, Nagtegaal ID, et al. An overview of 25 years of incidence, treatment and outcome of colorectal cancer patients. Int J Cancer 2018;143:2758-66.

3. Torky R, Alessa M, Kim HS, Sakr A, Zakarneh E, Sauri F, et al. Characteristics of patients presented with metastases during or after completion of chemoradiation therapy for locally advanced rectal cancer: a case series. Ann Coloproctol 2021;37:186-91.

4. van Gestel YR, Thomassen I, Lemmens VE, Pruijt JF, van HerkSukel MP, Rutten HJ, et al. Metachronous peritoneal carcinomatosis after curative treatment of colorectal cancer. Eur J Surg Oncol 2014;40:963-9.

5. Franko J, Shi Q, Meyers JP, Maughan TS, Adams RA, Seymour MT, et al. Prognosis of patients with peritoneal metastatic colorectal cancer given systemic therapy: an analysis of individual patient data from prospective randomised trials from the Analysis and 
Research in Cancers of the Digestive System (ARCAD) database. Lancet Oncol 2016;17:1709-19.

6. Lieu CH, Renfro LA, de Gramont A, Meyers JP, Maughan TS, Seymour MT, et al. Association of age with survival in patients with metastatic colorectal cancer: analysis from the ARCAD Clinical Trials Program. J Clin Oncol 2014;32:2975-84.

7. Doah KY, Shin US, Jeon BH, Cho SS, Moon SM. The impact of primary tumor resection on survival in asymptomatic colorectal cancer patients with unresectable metastases. Ann Coloproctol 2021;37:94-100.

8. Goodman MT, Shvetsov YB. Incidence of ovarian, peritoneal, and fallopian tube carcinomas in the United States, 1995-2004. Cancer Epidemiol Biomarkers Prev 2009;18:132-9.

9. Segelman J, Granath F, Holm T, Machado M, Mahteme H, Martling A. Incidence, prevalence and risk factors for peritoneal carcinomatosis from colorectal cancer. Br J Surg 2012;99:699-705.

10. Christou N, Auger C, Battu S, Lalloué F, Jauberteau-Marchan MO, Hervieu C, et al. Intraperitoneal chemotherapy for peritoneal metastases: technical innovations, preclinical and clinical advances and future perspectives. Biology (Basel) 2021;10:225.

11. Guinney J, Dienstmann R, Wang X, de Reyniès A, Schlicker A, Soneson C, et al. The consensus molecular subtypes of colorectal cancer. Nat Med 2015;21:1350-6.

12. Ubink I, van Eden WJ, Snaebjornsson P, Kok NF, van Kuik J, van Grevenstein WM, et al. Histopathological and molecular classification of colorectal cancer and corresponding peritoneal metastases. Br J Surg 2018;105:e204-11.

13. Sánchez-Hidalgo JM, Rodríguez-Ortiz L, Arjona-Sánchez Á, Rufián-Peña S, Casado-Adam Á, Cosano-Álvarez A, et al. Colorectal peritoneal metastases: optimal management review. World J Gastroenterol 2019;25:3484-502.

14. Bae SJ, Shin US, Ki YJ, Cho SS, Moon SM, Park SH. Role of peritoneal lavage cytology and prediction of prognosis and peritoneal recurrence after curative surgery for colorectal cancer. Ann Coloproctol 2014;30:266-73.

15. Dohan A, Hobeika C, Najah H, Pocard M, Rousset P, Eveno C. Preoperative assessment of peritoneal carcinomatosis of colorectal origin. J Visc Surg 2018;155:293-303.

16. Marin D, Catalano C, Baski M, Di Martino M, Geiger D, Di Giorgio $\mathrm{A}$, et al. 64-Section multi-detector row $\mathrm{CT}$ in the preoperative diagnosis of peritoneal carcinomatosis: correlation with histopathological findings. Abdom Imaging 2010;35:694-700.

17. van't Sant I, Engbersen MP, Bhairosing PA, Lambregts DM, BeetsTan RG, van Driel WJ, et al. Diagnostic performance of imaging for the detection of peritoneal metastases: a meta-analysis. Eur Radiol 2020;30:3101-12.

18. Pfannenberg C, Königsrainer I, Aschoff P, Oksüz MO, Zieker D, Beckert S, et al. (18)F-FDG-PET/CT to select patients with peritoneal carcinomatosis for cytoreductive surgery and hyperthermic intraperitoneal chemotherapy. Ann Surg Oncol 2009;16:1295303.

19. Iversen LH, Rasmussen PC, Laurberg S. Value of laparoscopy be- fore cytoreductive surgery and hyperthermic intraperitoneal chemotherapy for peritoneal carcinomatosis. Br J Surg 2013;100:28592.

20. Bereder JM, Classe JM, Ducreux M, Elias D, Ferron G, Gilly F, et al. Expert agreement on the minimal descriptive surgical report for peritoneal cancer. J Chir (Paris) 2007;144:463.

21. Liberale G, Vankerckhove S, Caldon MG, Ahmed B, Moreau M, Nakadi IE, et al. Fluorescence imaging after indocyanine green injection for detection of peritoneal metastases in patients undergoing cytoreductive surgery for peritoneal carcinomatosis from colorectal cancer: a pilot study. Ann Surg 2016;264:1110-5.

22. Harlaar NJ, Koller M, de Jongh SJ, van Leeuwen BL, Hemmer PH, Kruijff S, et al. Molecular fluorescence-guided surgery of peritoneal carcinomatosis of colorectal origin: a single-centre feasibility study. Lancet Gastroenterol Hepatol 2016;1:283-90.

23. Klaver YL, Simkens LH, Lemmens VE, Koopman M, Teerenstra S, Bleichrodt RP, et al. Outcomes of colorectal cancer patients with peritoneal carcinomatosis treated with chemotherapy with and without targeted therapy. Eur J Surg Oncol 2012;38:617-23.

24. Sugarbaker PH. Peritonectomy procedures. Ann Surg 1995;221: $29-42$.

25. Elias D, Blot F, El Otmany A, Antoun S, Lasser P, Boige V, et al. Curative treatment of peritoneal carcinomatosis arising from colorectal cancer by complete resection and intraperitoneal chemotherapy. Cancer 2001;92:71-6.

26. Shen P, Levine EA, Hall J, Case D, Russell G, Fleming R, et al. Factors predicting survival after intraperitoneal hyperthermic chemotherapy with mitomycin $\mathrm{C}$ after cytoreductive surgery for $\mathrm{pa}$ tients with peritoneal carcinomatosis. Arch Surg 2003;138:26-33.

27. Carboni F, Federici O, Sperduti I, Zazza S, Sergi D, Corona F, et al. Cytoreductive surgery with hyperthermic intraperitoneal chemotherapy for peritoneal carcinomatosis from epithelial ovarian cancer: a 20-year single-center experience. Cancers (Basel) 2021; 13:523.

28. Roh SJ, Park SC, Choi J, Lee JS, Lee DW, Hong CW, et al. Cytoreductive surgery and hyperthermic intraperitoneal chemotherapy with mitomycin $\mathrm{C}$ used for colorectal peritoneal carcinomatosis. Ann Coloproctol 2020;36:22-9.

29. Kim WR, Hur H, Min BS, Baik SH, Lee KY, Kim NK. Single center experience with hyperthermic intraperitoneal chemotherapy. Ann Coloproctol 2017;33:16-22.

30. Verwaal VJ, van Ruth S, de Bree E, van Sloothen GW, van Tinteren $\mathrm{H}$, Boot H, et al. Randomized trial of cytoreduction and hyperthermic intraperitoneal chemotherapy versus systemic chemotherapy and palliative surgery in patients with peritoneal carcinomatosis of colorectal cancer. J Clin Oncol 2003;21:3737-43.

31. Verwaal VJ, Bruin S, Boot H, van Slooten G, van Tinteren H. 8-year follow-up of randomized trial: cytoreduction and hyperthermic intraperitoneal chemotherapy versus systemic chemotherapy in patients with peritoneal carcinomatosis of colorectal cancer. Ann Surg Oncol 2008;15:2426-32.

32. Elias D, Lefevre JH, Chevalier J, Brouquet A, Marchal F, Classe 
JM, et al. Complete cytoreductive surgery plus intraperitoneal chemohyperthermia with oxaliplatin for peritoneal carcinomatosis of colorectal origin. J Clin Oncol 2009;27:681-5.

33. Franko J, Ibrahim Z, Gusani NJ, Holtzman MP, Bartlett DL, Zeh HJ 3rd. Cytoreductive surgery and hyperthermic intraperitoneal chemoperfusion versus systemic chemotherapy alone for colorectal peritoneal carcinomatosis. Cancer 2010;116:3756-62.

34. Cashin PH, Mahteme H, Spång N, Syk I, Frödin JE, Torkzad M, et al. Cytoreductive surgery and intraperitoneal chemotherapy versus systemic chemotherapy for colorectal peritoneal metastases: a randomised trial. Eur J Cancer 2016;53:155-62.

35. Quénet F, Elias D, Roca L, Goéré D, Ghouti L, Pocard M, et al. Cytoreductive surgery plus hyperthermic intraperitoneal chemotherapy versus cytoreductive surgery alone for colorectal peritoneal metastases (PRODIGE 7): a multicentre, randomised, openlabel, phase 3 trial. Lancet Oncol 2021;22:256-66.

36. Song N, Pogue-Geile KL, Gavin PG, Yothers G, Kim SR, Johnson $\mathrm{NL}$, et al. Clinical outcome from oxaliplatin treatment in stage II/ III colon cancer according to intrinsic subtypes: secondary analysis of NSABP C-07/NRG oncology randomized clinical trial. JAMA Oncol 2016;2:1162-9.

37. Reymond MA, Hu B, Garcia A, Reck T, Köckerling F, Hess J, Morel P. Feasibility of therapeutic pneumoperitoneum in a large animal model using a microvaporisator. Surg Endosc 2000;14:51-5.

38. Solaß W, Hetzel A, Nadiradze G, Sagynaliev E, Reymond MA. Description of a novel approach for intraperitoneal drug delivery and the related device. Surg Endosc 2012;26:1849-55.

39. Solass W, Herbette A, Schwarz T, Hetzel A, Sun JS, Dutreix M, et al. Therapeutic approach of human peritoneal carcinomatosis with Dbait in combination with capnoperitoneum: proof of concept. Surg Endosc 2012;26:847-52.

40. Klaver CE, Stam R, Sloothaak DA, Crezee J, Bemelman WA, Punt $\mathrm{CJ}$, et al. Colorectal cancer at high risk of peritoneal metastases: long term outcomes of a pilot study on adjuvant laparoscopic HIPEC and future perspectives. Oncotarget 2017;8:51200-9.

41. Klaver CE, Wisselink DD, Punt CJ, Snaebjornsson P, Crezee J, Aalbers AG, et al. Adjuvant hyperthermic intraperitoneal chemotherapy in patients with locally advanced colon cancer (COLOPEC): a multicentre, open-label, randomised trial. Lancet Gastroenterol Hepatol 2019;4:761-70.

42. Goéré D, Glehen O, Quenet F, Guilloit JM, Bereder JM, Lorimier $\mathrm{G}$, et al. Second-look surgery plus hyperthermic intraperitoneal chemotherapy versus surveillance in patients at high risk of developing colorectal peritoneal metastases (PROPHYLOCHIPPRODIGE 15): a randomised, phase 3 study. Lancet Oncol 2020; 21:1147-54.

43. Jacquet P, Sugarbaker PH. Clinical research methodologies in diagnosis and staging of patients with peritoneal carcinomatosis. Cancer Treat Res 1996;82:359-74.

44. Sugarbaker PH. Cytoreductive surgery and peri-operative intraperitoneal chemotherapy as a curative approach to pseudomyxoma peritonei syndrome. Eur J Surg Oncol 2001;27:239-43.
45. Yurttas C, Hoffmann G, Tolios A, Haen SP, Schwab M, Königsrainer I, et al. Systematic review of variations in hyperthermic intraperitoneal chemotherapy (HIPEC) for peritoneal metastasis from colorectal cancer. J Clin Med 2018;7:567.

46. Mahteme H, Hansson J, Berglund A, Påhlman L, Glimelius B, Nygren $\mathrm{P}$, et al. Improved survival in patients with peritoneal metastases from colorectal cancer: a preliminary study. Br J Cancer 2004;90: 403-7.

47. Glehen O, Kwiatkowski F, Sugarbaker PH, Elias D, Levine EA, De Simone M, et al. Cytoreductive surgery combined with perioperative intraperitoneal chemotherapy for the management of peritoneal carcinomatosis from colorectal cancer: a multi-institutional study. J Clin Oncol 2004;22:3284-92.

48. Sugarbaker PH, Gianola FJ, Speyer JC, Wesley R, Barofsky I, Meyers CE. Prospective, randomized trial of intravenous versus intraperitoneal 5-fluorouracil in patients with advanced primary colon or rectal cancer. Surgery 1985;98:414-22.

49. Chua TC, Liauw W, Zhao J, Morris DL. Comparative analysis of perioperative intraperitoneal chemotherapy regimen in appendiceal and colorectal peritoneal carcinomatosis. Int J Clin Oncol 2013;18:439-46.

50. Elias D, Benizri E, Di Pietrantonio D, Menegon P, Malka D, Raynard B. Comparison of two kinds of intraperitoneal chemotherapy following complete cytoreductive surgery of colorectal peritoneal carcinomatosis. Ann Surg Oncol 2007;14:509-14.

51. Cashin PH, Graf W, Nygren P, Mahteme H. Intraoperative hyperthermic versus postoperative normothermic intraperitoneal chemotherapy for colonic peritoneal carcinomatosis: a case-control study. Ann Oncol 2012;23:647-52.

52. Klaver YL, Hendriks T, Lomme RM, Rutten HJ, Bleichrodt RP, de Hingh IH. Hyperthermia and intraperitoneal chemotherapy for the treatment of peritoneal carcinomatosis: an experimental study. Ann Surg 2011;254:125-30.

53. Rossi AJ, Khan TM, Rehman SU, Nash GM, Hernandez JM. Early postoperative intraperitoneal versus hyperthermic intraperitoneal chemotherapy after optimal cytoreductive surgery for colorectal cancer with isolated peritoneal metastasis (ICARuS). Ann Surg Oncol 2021;28:4100-1.

54. Eveno C, Passot G, Goéré D, Soyer P, Gayat E, Glehen O, et al. Bevacizumab doubles the early postoperative complication rate after cytoreductive surgery with hyperthermic intraperitoneal chemotherapy (HIPEC) for peritoneal carcinomatosis of colorectal origin. Ann Surg Oncol 2014;21:1792-800.

55. Han MH, Park YY, Pratap S, Han YD, Cho MS, Hur H, et al. Shortterm outcomes after upfront chemotherapy followed by curative surgery in metastatic colon cancer: a comparison with upfront surgery patients. Ann Coloproctol 2019;35:327-34.

56. Rovers KP, Simkens GA, Punt CJ, van Dieren S, Tanis PJ, de Hingh IH. Perioperative systemic therapy for resectable colorectal peritoneal metastases: Sufficient evidence for its widespread use?: a critical systematic review. Crit Rev Oncol Hematol 2017;114:5362. 
57. Waite K, Youssef H. The role of neoadjuvant and adjuvant systemic chemotherapy with cytoreductive surgery and heated intraperitoneal chemotherapy for colorectal peritoneal metastases: a systematic review. Ann Surg Oncol 2017;24:705-20.

58. Glockzin G, Zeman F, Croner RS, Königsrainer A, Pelz J, Ströhlein MA, et al. Perioperative systemic chemotherapy, cytoreductive surgery, and hyperthermic intraperitoneal chemotherapy in patients with colorectal peritoneal metastasis: results of the prospective multicenter phase 2 COMBATAC trial. Clin Colorectal Cancer 2018;17:285-96.

59. Rovers KP, Bakkers C, Nienhuijs SW, Burger JW, Creemers GM, Thijs AM, et al. Perioperative systemic therapy vs cytoreductive surgery and hyperthermic intraperitoneal chemotherapy alone for resectable colorectal peritoneal metastases: a phase 2 randomized clinical trial. JAMA Surg 2021;156:710-20.

60. Passot G, Bakrin N, Garnier L, Roux A, Vaudoyer D, Wallet F, et al. Intraperitoneal vascular endothelial growth factor burden in peritoneal surface malignancies treated with curative intent: the first step before intraperitoneal anti-vascular endothelial growth factor treatment? Eur J Cancer 2014;50:722-30.

61. Lu CS, Lin JK, Chen WS, Lin TC, Jiang JK, Yang SH, et al. Intraperitoneal ziv-aflibercept effectively manages refractory ascites in colorectal cancer patients. Oncotarget 2017;8:36707-15.

62. Seimetz D. Novel monoclonal antibodies for cancer treatment: the trifunctional antibody catumaxomab (removab). J Cancer 2011; 2:309-16. 